\title{
On the $\mathrm{YFe}_{11} \mathrm{Mo}$ intermetallic characterization
}

\author{
D. Nunes*, A.P. Gonçalves**, J.Th.M. De Hosson*** and P.A. Carvalho*
}

* ICEMS, Instituto Superior Técnico, University of Lisbon, Av. Rovisco Pais, 1049-001 Lisboa, Portugal

**IST/ITN, Instituto Superior Técnico, Unversity of Lisbon and CFMC-UL, Estrada Nacional 10, 2686-953 Sacavém, Portugal

***Department of Applied Physics, University of Groningen, Nijenborgh 4, 9747 AG Groningen, The Netherlands

Rare-earth intermetallic compounds adopting the tetragonal $\mathrm{ThMn}_{12}$-type structure and containing high $\mathrm{Fe}$ concentrations have attracted considerable attention in the field of permanent magnets [1]. Among them, the Y-Fe-Mo series has been extensively investigated [2,3], especially by X-ray diffraction (XRD), but the microstructural characterization was very limited.

In the present work, Y:11Fe:Mo has been prepared by melting Y, Fe and Mo in an arc furnace followed by splatquenching and/or annealing treatments. The structure, chemistry and magnetic domain configurations of the resulting polycrystalline aggregates have been investigated by XRD, scanning and transmission electron microscopies (SEM and TEM, respectively), energy dispersive X-ray spectroscopy (EDS) and Lorentz microscopy.

A special emphasis was given to Lorentz microscopy, where contrast is based on the Lorentz deflection imposed on electrons by the passage through a magnetic specimen. The magnetic domains have been imaged by the intermediate lens in Fresnel mode, with the objective lens switched off. In these conditions the intermediate lens is defocused so that out-of-focus images of the specimen are formed: the magnetic domain walls are imaged as alternate bright (convergent) and dark (divergent) lines. For the overfocused image, bright lines occur at the position of domain walls for which the magnetisation on either side is deflecting the electrons towards the wall, whereas dark lines are observed at the walls for which the magnetisation on either side is deflecting the electrons away from the wall. The opposite contrast is observed at the underfocused image.

The results have shown that the tetragonal $\mathrm{YFe}_{11-\mathrm{x}} \mathrm{Mo}_{\mathrm{y}}$ phase is predominant, with a cellular dendritic morphology (Figure 1), but a considerable presence of $\alpha-\mathrm{Fe}(\mathrm{Mo})$ could be inferred [4], forming a coarse intercellular eutectic mixture. Significant Fe segregation occurred during annealing. However, this composition variation corresponded to an extremely limited evolution of the lattice parameters, the Rietveld analysis pointing to Fe vacancies at the $8 i$ sites on the annealed material. XRD and EDS results indicate that the fraction of point defects in the ThMn $\mathrm{Mn}_{12}$-type structure adapts to the processing route and that the stable configuration depends on the temperature. The current study also showed that grain boundaries are usually associated with domain walls and that $\mathrm{YFe}_{11} \mathrm{Mo}$ grains present internal domain walls forming stripe/maze patterns characteristic of high anisotropy materials (Figure 2), while residual $\alpha$ $\mathrm{Fe}(\mathrm{Mo})$ grains exhibit vortex configurations (see arrows in Figures $2(\mathrm{a}-\mathrm{c}))$ [5].

\section{References}

1. Nunes D. et al., Appl. Phys. A, 104:1053, 2011.

2. Müller A.J., Appl. Phys., 64:249, 1988.

3. Mao W. et al., J. Phys. Condens. Matter., 10:4379, 1998.

4. Nunes D. et al., Mater. Charact., 60:1607, 2009.

5. Nunes D. et al., J. Alloys Compd. 487:11, 2009.

The work was supported by the Portuguese Science Foundation through the CTM/48617/2002 and PEst-OE/CTMUI0084/2011 grants. 

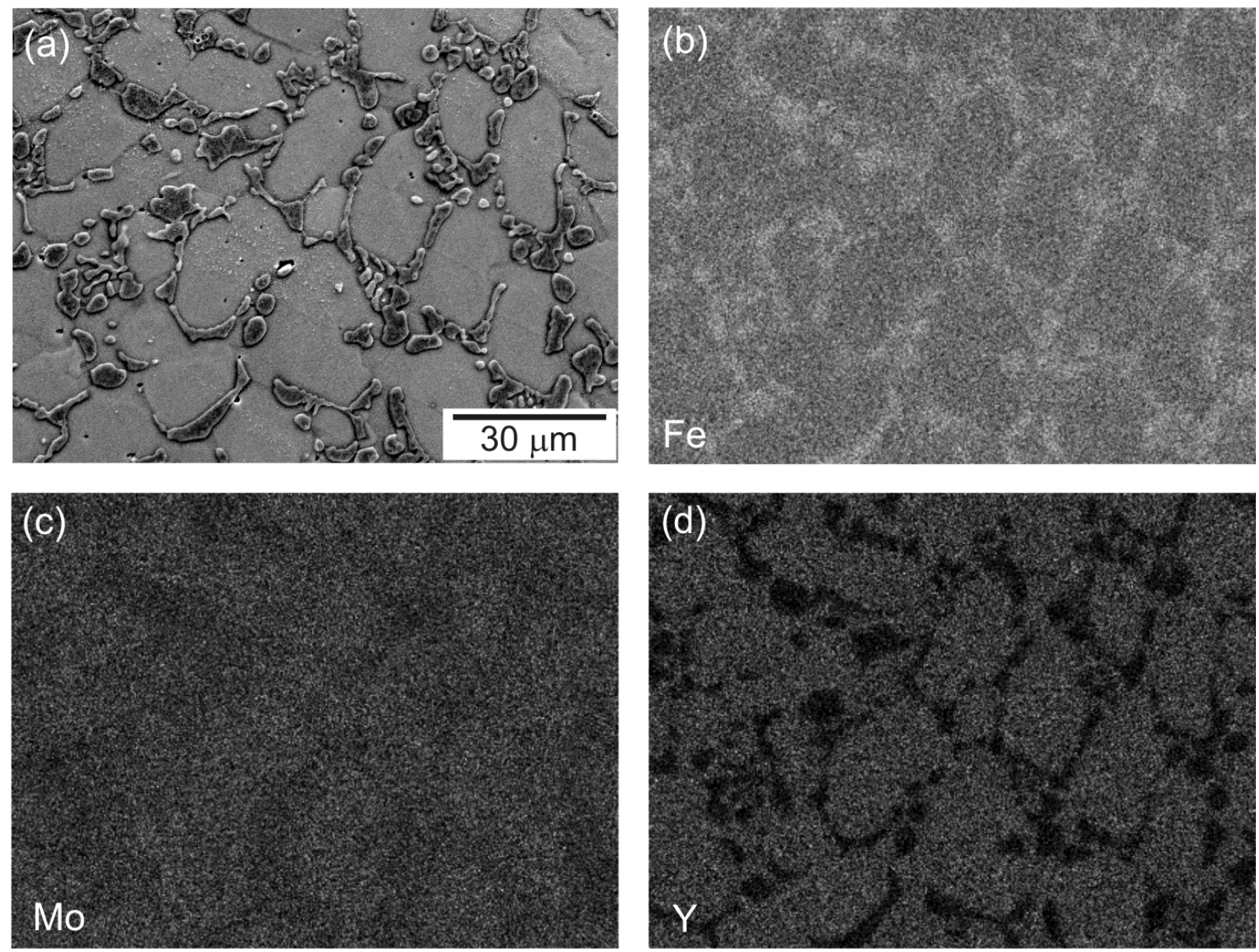

Figure 1. Y:11Fe:Mo alloy under the as-cast condition (etched sample). (a) Secondary electron SEM image of the corresponding X-ray maps for Fe (b), Mo (c) and Y (d). EDS point analyses showed that the cellular structure corresponds to a primary crystallization of $\mathrm{YFe}_{11-\mathrm{x}} \mathrm{Mo}_{\mathrm{y}}$ with a $\alpha-\mathrm{Fe}(\mathrm{Mo})+\mathrm{YFe}_{11-\mathrm{x}} \mathrm{Mo}_{\mathrm{y}}$ eutectic mixture at the intercellular regions.
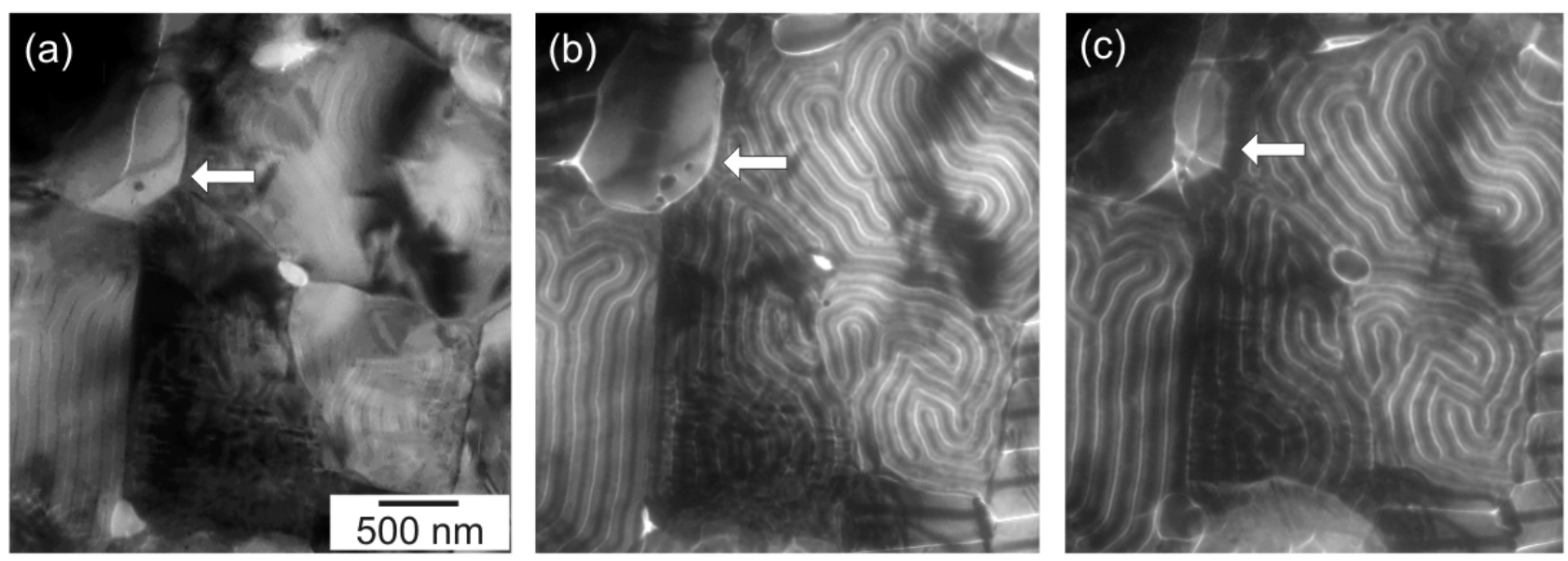

Figure 2. Microstructural and domain configuration of the Y:11Fe:Mo splat-quenched/annealed material, (a) in focus: $\Delta f=0 \mathrm{~mm}$, (b) overfocused: $\Delta f=+196 \mathrm{~mm}$, and (c) underfocused: $\Delta f=-208 \mathrm{~mm}$. The arrows point to vortex magnetic configurations in $\alpha-\mathrm{Fe}(\mathrm{Mo})$ grains. The dark lines in between are also walls. The work was supported by the Portuguese Science Foundation through the CTM/486 